\title{
Pimenta pseudocaryophyllus (Gomes) Landrum: aspectos botânicos, ecológicos, etnobotânicos e farmacológicos
}

D'ANGELIS, A.S.R. ${ }^{1}$; NEGRELLE, R.R.B. ${ }^{2}$

'Universidade Federal do Paraná, Programa de Pós Graduação em Agronomia, Produção Vegetal, Rua dos Funcionários, 1540, Juvevê, Curitiba, PR, CEP: 80035-050 *amandadangelis@ufpr.br ${ }^{2}$ Laboratório Oikos de Ecologia, Departamento de Botânica, Centro Politécnico, Avenida Coronel Francisco Heráclito dos Santos, 210, Jardim das Américas, Curitiba, PR, CEP: 81531-970.

RESUMO: Pimenta pseudocaryophyllus (Gomes) Landrum é a única espécie representante brasileira deste gênero de Myrtaceae. Tem sido popularmente utilizada no tratamento de diversas enfermidades, assim como condimento e aromatizante de bebidas, por seu sabor muito similar ao cravo-da-índia. Em menor escala, é empregada em carpintaria e na arborização urbana. Diversos autores analisaram a composição química do óleo essencial da espécie em diferentes regiões do Brasil, encontrando eugenol, metileugenol, (E) metilisoeugenol, chavibetol, geranial e neral como composto predominante nos espécimes estudados. Visando ressaltar a importância de $P$. pseudocaryophyllus como potencial fonte de recursos assim como subsidiar ações de manejo adequado esta revisão apresenta os principais aspectos botânicos, ecológicos, etnobotânicos e farmacológicos da espécie.

Palavras-chave: Pimenta pseudocaryophyllus, cataia, produto florestal não madeirável, etnobotânica, atividades farmacológicas

\begin{abstract}
Pimenta pseudocaryophyllus (Gomes) Landrum: botanical, ecological, ethnobotanical and pharmacological aspects. Pimenta pseudocaryophyllus (Gomes) Landrum is the only Brazilian species representative of this Myrtaceae genus. It has being traditionally used in the treatment of many diseases as well for seasoning food and flavoring beverages due to its similarity with clove flavor. In a minor scale, it is used in carpentry and as urban tree. Many authors have analyzed the chemical composition of the leaf essential oil in different regions of Brazil, founding eugenol, metileugenol, (E) metilisoeugenol, chavibetol, geranial e neral as main component in the specimens studied. Aiming to highlight the importance of $P$. pseudocaryophyllus as potential source of natural products and to provide some base to the adequate management actions, a review on botanical, ecological, ethnobotanical and pharmacological aspects of this species is presented.
\end{abstract}

Keywords: Pimenta pseudocaryophyllus, cataia, non-timber forest product, ethnobotany, pharmacological activities

\section{INTRODUÇÃO}

A alta diversidade cultural e biológica que permeiam nosso país estão indissociavelmente relacionadas. O Brasil detém $22 \%$ de todas as espécies de plantas descritas no mundo, e milhares de comunidades quilombolas, de pescadores artesanais, agricultores familiares, sertanejos, ribeirinho, etc.

Esse patrimônio biológico é fonte de recursos materiais genéticos, simbólicos e econômicos para esses povos que, por sua vez, são detentores de rico conhecimento referente ao mundo natural (Bandeira, 2010).

Durante muitos séculos as plantas medicinais consistiram na base da terapêutica. Sociedades tradicionais possuem uma vasta farmacopeia natural, em boa parte proveniente dos recursos vegetais encontrados nos ambientes naturais ocupados por estas populações (Amorozo, 2002). Por volta do século XIX, com o advento da pesquisa científica, muitas substâncias puderam ser isoladas das plantas e serviram de modelos para a produção de fármacos, o que hoje representa proporção substancial do mercado global de medicamentos (Yunes \& Cechinel Filho, 2001).

Nesse sentido se dá a dimensão da importância dos estudos dos significados culturais

Recebido para publicação em 09/04/2013

Aceito para publicação em 24/03/2014

10.1590/1983-084X/13 026

Rev. Bras. PI. Med., Campinas, v.16, n.3, p.607-617, 2014. 
das plantas, da diversidade de uso dos recursos entre populações, e dos fatores socioculturais que se relacionam com esse conhecimento (Bandeira, 2010). Constata-se que a base empírica desenvolvida pelas populações tradicionais ao longo do tempo pode, em muitos casos, ter uma comprovação científica, que habilitaria a extensão destes usos à sociedade industrializada (Farnsworth, 1988). A transmissão desse conhecimento, bem como pesquisas acerca das atividades farmacológicas e toxicológicas das plantas, vem como reforço à preservação de inúmeras espécies vegetais e à utilização racional, eficaz e segura dos fitoterápicos (Agra, 1982; Yunes \& Cechinel Filho, 2001).

A espécie Pimenta pseudocaryophyllus (Gomes) L. R. Landrum tem sido amplamente utilizada na medicina tradicional para o tratamento de diversas enfermidades, além de estar vinculada a outras práticas tradicionais, como o uso de seus frutos para condimentar alimentos e da madeira para obras internas de carpintaria (Lullez, 1991; Nakaoka-sakita et. al., 1994; Lorenzi, 1998; Paula, 2008; Farias, 2009; Morgante et al., 2010).

Outro uso importante da espécie, que teve início na década de 80 , foi a saborização de aguardente com folhas da planta, hoje comercializada ao longo do litoral dos estados do Paraná e São Paulo. Em geral, o uso da espécie é feito a partir de ação extrativista em remanescentes florestais onde ocorre espontaneamente, fato que pode comprometer a dinâmica populacional e a manutenção do estoque natural da espécie, além da qualidade do produto, já que a composição química é influenciada pelas variações climáticas e edáficas (Morgante et al., 2010).

Encontra-se na literatura científica diversos registros de aplicações para o óleo essencial da espécie, entre elas, em aromatizantes, cosméticos, perfumes e inseticidas e na indústria fármacomedicinal (Farias, 2009). Estudos iniciais têm sugerido o potencial farmacológico para a espécie e indicam serem os componentes do óleo essencial os responsáveis por este potencial (Lima et al., 2006; Paula et al., 2008; Custódio et al., 2010; Fajemiroye, 2012).

Considerando o potencial terapêutico de $P$. pseudocaryophyllus e o crescente interesse econômico pela espécie, o presente trabalho teve como objetivo revisar os principais aspectos botânicos, ecológicos, etnobotânicos e farmacológicos descritos na literatura. A sistematização do conhecimento a respeito da espécie pretende valorizar e preservar os conhecimentos tradicionais que, aliados ao conhecimento científico, possam promover o uso racional da espécie como recurso econômico e terapêutico.

\section{Fonte de dados}

Para a realização desse trabalho buscouse artigos originais e revisões indexados nas bases Scielo, Periódicos Capes e ISI Web of Knowledge. Para a busca foram usadas as palavras-chave Pimenta e Pimenta pseudocaryophyllus. Utilizaramse, ainda, livros nacionais e internacionais de botânica, ecologia, farmacologia de produtos naturais e etnobotânica. Não houve restrição quanto ao ano de publicação.

\section{Caracterização taxonômica}

A família Myrtaceae compreende cerca de 130 gêneros e 4000 espécies de árvores e arbustos que se distribuem por todos os continentes, com exceção da Antártica, mas com nítida predominância nas regiões tropicais e subtropicais do mundo, representando uma das maiores famílias da flora brasileira (Souza \& Lorenzi, 2005; Gressler et al., 2006).

Esta é uma das famílias botânicas mais importantes do Brasil (Paula et al., 2010a), representando o componente lenhoso dominante em várias formações vegetais brasileiras, especialmente a Floresta Atlântica (Gressler et al., 2006).

Myrtaceae destaca-se também entre os representantes da flora brasileira por apresentar grande número de espécies de interesse medicinal, sendo reconhecida por seu grande potencial na produção de óleos voláteis de importância econômica (Lima et al., 2006). Dentre os representantes de interesse farmacológico da família, destacam-se as espécies do gênero Pimenta, conhecidas especialmente pela importância econômica atribuída a duas delas, $P$. dioica (L.) Merrill (allspice) e P. racemosa (Miller) J. Moore (bay tree). A primeira é utilizada principalmente como condimento ou especiaria, sendo também utilizada no tratamento de doenças pelas populações da América Central e do Caribe. Também é um dos ingredientes básicos de uma bebida denominada Pru muito popular em Cuba, à qual são atribuídas propriedades refrescantes e medicinais. Por sua vez, $P$. racemosa é utilizada na medicina popular para o tratamento de reumatismo, dor de dente, dores abdominais, febre, pneumonia e gripe (Paula et al., 2010a).

O gênero Pimenta foi descrito por John Lindley em 1821 (Collectanea Botanica 4: sub t. 19. 1821), sendo $P$. officinalis a espécie typus. A denominação deste gênero vem do latim pigmentum, "corante", do verbo pingere, "colorir, pintar, bordar", em associação com as características do fruto daquela espécie. Neste caso, o nome atendeu mais aos aspectos visuais do que aos do sabor acrescentado pelo fruto à comida (Weiss, 2002). Possui 15 espécies distribuídas na América, 
com maioria localizada no Caribe (Mazine, 2008; Landrum, 1986). No Brasil, este gênero esta representado apenas por P. pseudocaryophyllus (Gomes) L.R. Landrum (Farias et al., 2009).

Esta espécie foi descrita inicialmente, em 1812, como Myrtus pseudocaryophyllus pelo médico e botânico português Bernardino A. Gomes, que estudou várias plantas brasileiras. Em 1984, a espécie foi reposicionada no gênero Pimenta pelo botânico norte-americano Leslie R. Landrum.

Em termos taxonômicos, no sistema APG II, P. pseudocaryophyllus está categorizada em:

Ordem Myrtales

Família Myrtaceae Juss.

Subfamília Myrtoidea

Tribo Myrtaea

Gênero Pimenta Lindl.

Espécie Pimenta pseudocaryophyllus (Gomes) Landrum

(Souza \& Lorenzi, 2005).

A espécie é separada em três variedades regionais (LANDRUM, 1986):

P. pseudocaryophyllus var. hoehnei (DC.) Landrum

P. pseudocaryophyllus var. fulvescens (Burret) Landrum

P. pseudocaryophyllus var. pseudocaryohyllus (Gomes) Landrum

Os extremos dessas variedades são prontamente distinguíveis, entretanto, existem numerosos intermediários.

P. pseudocaryophyllus var. pseudocaryophyllus é uma árvore de pequeno porte típica de florestas e áreas montanhosas do sudeste do Brasil. P. pseudocaryophyllus var. fulvescens (A. $P$. de Canolle) Landrum é uma pequena árvore ou arbusto que se localiza em regiões secas no centro sul do Brasil, com alguns exemplares na Bolívia. P. pseudocaryophyllus var. hoehnei (Burret) Landrum é uma pequena árvore confinada na região florestal litorânea do sudeste do Brasil, de Santa Catarina a São Paulo. As três variedades diferenciam-se especialmente pela dimensão das folhas e do pecíolo, sendo as maiores medidas encontradas em Pimenta pseudocaryophyllus var. fulvescens, e as menores em Pimenta pseudocaryophyllus var. hoehnei. Diferenciam-se, ainda, pelo número de flores nas inflorescências, sendo que nas variedades pseudocaryophyllus e fulvescens as inflorescências apresentam-se em dicásio ou panícula de, usualmente, sete a quinze flores, enquanto em hoehnei as inflorescências apresentam-se em dicásio com, no máximo, três flores (Landrum, 1986).

Recentemente, baseado em coletas realizadas ao longo de mais de vinte anos que sucederam a revisão de Landrum, levantou-se a questão de que a variação morfológica possa compreender, na verdade, mais de uma espécie brasileira de pimenta (Morgante et al., 2010).

Sinonímia (Missouri Botanical Garden, 2012):

Eugenia acuminata Link

Eugenia fulvescens A.DC

Eugenia leandreana O.Berg

Eugenia mutabilis O.Berg

Eugenia pseudocaryophyllus (Gomes) DC.

Myrtus fulvescens (DC.) Kiaersk.

Myrtus pseudocaryophyllus Gomes

Myrtus velutina (O. Berg) Kiaersk.

Myrtus velutina fo. macrophylla Kiaersk.

Pseudocaryophyllus sericeus O. Berg

Pseudocaryophyllus costatus O.Berg

Pseudocaryophyllus acuminatus (Link) Burret

Pseudocaryophyllus organensis Burret

Pseudocaryophyllusglaziovianus (Kiaersk.) Burret

Pseudocaryophyllus chrysophyllus Burret 


\author{
Pseudocaryophyllus platyphyllus Burret \\ Pseudocaryophyllus jaccoudii Mattos \\ Pseudocaryophyllusfulvescens (A.DC.) O. Berg \\ Pseudocaryophyllus velutinus O.Berg \\ Pseudocaryophyllus \\ mutabilis (O.Berg) Burret \\ Pseudocaryophyllus hoehnei Burret \\ Pseudocaryophyllus emarginatus Burret \\ Pseudocaryophyllus theifer Toledo
}

Nomes populares (Nakaoka-sakita et al., 1994; Lorenzi, 1998; Girard, 2005; Sakita \& Aguiar, 2007; Paula et al., 2008; Aciole, 2009; Farias et al., 2009; Morgante et al., 2010; Oliveira, 2010):

Cataia ("folha que queima" em tupi-guarani) - litoral do Paraná e Registro, no Vale do Ribeira (SP); chá-de-bugre, craveiro-do-mato, louro, lourocravo, pau-cravo - Paraná e São Paulo; casca rosa, limão do mato - Goiás; chá-da-terra, cravo-da-terra, louro-da-terra, louro-falso, falso-cravo - São Paulo; craveiro - Paraná.

\section{Descrição botânica}

$P$. pseudocaryophyllus é uma planta aromática, de 4 a $10 \mathrm{~m}$ de altura, dotada de copa arredondada muito característica. Em algumas regiões pode ocorrer como arbusto. O tronco geralmente ereto com casca fissurada, em vista frontal apresenta coloração que vai do acastanhado ao acinzentado. Possui inflorescências axilares em panículas ou dicásios, com 7 a 15 flores bissexuais, com estames numerosos e de cor branca. Seu fruto é uma baga subglobosa, de polpa suculenta, contendo de uma a duas sementes duras. Os frutos, quando maduros, adquirem coloração muito escura, quase negra (Brandão, 2002; Lorenzi, 2002; Paula et al., 2008; Morgante et al., 2010). É a única espécie pertencente às Myrtaceae que combina embrião espiralizado e intumescido com capa de semente dura (Paula et al., 2010a).

A filotaxia é oposta-cruzada. As folhas são simples, inteiras, curtamente pecioladas, peninérveas e de consistência coriácea; glabras na superfície adaxial e pilosas na superfície abaxial. Lâmina de coloração verde-escura na face superior e verde-prateada na face inferior, com 10 a $17 \mathrm{~cm}$ de comprimento e 3 a $5 \mathrm{~cm}$ de largura. A lâmina é lanceolada com as margens levemente onduladas, o ápice é agudo e a base acunheada (Farias et al., 2009). Quando verificadas contra a luz, apresentam vários pontos transparentes espalhados, caracterizando a presença de cavidades secretoras de substâncias terpênicas dispostas ao longo do mesofilo que quando amassadas entre os dedos liberam um odor característico (Paula et al., 2008). Morfo-anatomicamente, a lâmina foliar de
P.pseudocaryophyllus apresenta características frequentemente observadas em Myrtaceae, com predominância de cavidades secretoras, complexos estomáticos anomocíticos ou paracíticos, além da presença de numerosos idioblastos portadores de drusas. O mesofilo está representado pelos parênquimas paliçádico e lacunoso. O parênquima paliçádico é formado por células alongadas compactamente dispostas em duas ou três camadas. O parênquima lacunoso é formado por células globosas frouxamente distribuídas em aproximadamente seis camadas. Ao longo do mesofilo, principalmente na região do parênquima lacunoso, há uma enorme quantidade de idioblastos portadores de drusas e cristais prismáticos.

Apresenta lâminas foliares dorsiventrais e hipoestomáticas, com estômatos predominantemente anomocíticos. Há grande quantidade de tricomas unicelulares alongados na superfície abaxial. As paredes anticlinais das células da epiderme abaxial, em vista frontal, apresentam-se espessas e sinuosas. Há cavidades secretoras contendo compostos lipídicos que se exteriorizam para as duas epidermes, porém são mais abundantes na superfície adaxial

Farias et al. (2009), estudaram a organização estrutural da folha de espécimes coletados em um fragmento de Floresta Ombrófila Mista, no Paraná. Observou-se que as células estomáticas apresentam-se em posição elevada em relação às demais células epidérmicas, diferindo da maioria dos representantes de Myrtales, que possuem estômatos nivelados com as demais células. Essa característica deve estar relacionada ao fato de a espécie se desenvolver em locais sombreados, recebendo baixa intensidade luminosa.

Em espécimes coletados em Minas Gerais e no Distrito Federal, Paula et al., (2008), o corte transversal da região internervural revelou epidermes adaxial e abaxial uniestratificadas, recobertas por grossa cutícula, hipoderme com duas a três camadas, parênquima paliçádico compacto, com duas ou três camadas, e parênquima lacunoso com espaços conspícuos formado por até seis camadas. Tais características são consideradas 
xeromórficas, ou seja, adaptações fisiológicas à alta intensidade luminosa.

\section{Distribuição e ocorrência}

P. pseudocaryophyllus ocorre principalmente nas regiões montanhosas e costeiras do Sul e Sudeste do Brasil, tendo sido registrada em Minas Gerais, Espírito Santo, São Paulo, Rio de Janeiro, Paraná, Santa Catarina, Rio Grande do Sul e Distrito Federal (Paula et al., 2008; Sobral, 2010).

Esta espécie é característica do Domínio da Mata Atlântica incluindo Floresta Ombrófila Densa, Floresta Estacional Semidecidual e Restinga (Sobral, 2010). Nesses ambientes geralmente apresenta vasta, expressiva, porém descontínua dispersão. Raramente ocorre na Floresta Ombrófila Mista Montana (Legrand \& Klein, 1978). Ocorre também, de forma rara, em regiões de Cerrado brasileiro, especialmente cerradão (Paula et al., 2008).

As formas que ocorrem na Caatinga da Bahia e na Mata Atlântica do alto do Serra do Mar desde o Estado de São Paulo até o Estado de Santa Catarina são consideradas variedades distintas (Landrum, 1986; Lorenzi, 1998; Brandão, 2002).

\section{Aspectos ecológicos a agronômicos}

Espécie semidecídua, heliófita, seletiva xerófila e pioneira. Pouco exigente quanto à qualidade do solo, geralmente ocorre como população homogênea em solos pobres, bem arenosos e drenados (Lorenzi, 1998). No sul do Brasil é típica da vegetação das partes elevadas da Serra do Mar, comportando-se como espécie esciófila, e hidrófila, ocorrendo, principalmente, nas encostas rochosas de solos pouco profundos do alto dos morros, em matas pouco densas e baixas (Legrand \& Klein, 1978).

No que se refere à estrutura populacional, registrou-se no município de Tijucas do Sul (PR), o padrão "J invertido", indicando predominância de indivíduos das fases iniciais de desenvolvimento em detrimento de fases mais avançadas (Péllico Neto, 2007). Em termos genéticos, detectou-se elevado polimorfismo para esta espécie (Sebastião et al., 2011).

Floresce em dezembro e janeiro e frutifica de maio a julho (Lorenzi, 1998; Brandão, 2002). No Parque Estadual da Ilha do Cardoso, município de Cananéia/SP, observou-se botão floral de setembro a outubro; flor em outubro; fruto verde de novembro a janeiro e fruto maduro de janeiro a fevereiro (Staggemeier, 2007).

$P$. pseudocaryophyllus reproduz-se por sementes, desconhecendo-se outras formas de propagação (Ruschel, 2011). Produz anualmente moderada quantidade de sementes viáveis (Brandão,
2002). Os frutos são muito procurados por pássaros (Lorenzi, 1998; Ruschel, 2011).

Em ambiente aberto com sombreamento $50 \%$, empregando-se substrato misto (Plantmax e vermiculita, 3:1) com 0,5 g.L-1 de NPK 10.10 .10 e 2,0 g.L-1 de calcário dolomítico, registrou-se emergência após 15 dias da semeadura, finalizando-se a emergência após 22 dias. A taxa de germinação foi de $79 \%$, sendo considerada alta (Morgante et al., 2010).

P. pseudocaryophyllus caracteriza-se por apresentar emergência epígea desuniforme, ou seja, distribuída ao longo de vários dias. A emergência inicial é curvada. O cotilédone é foliáceo, séssil, com limbo inteiro. Os cotilédones emergem verdes com a superfície inferior vinácea, e tornam-se completamente verdes; apresentam grande quantidade de glândulas de óleo essencial, sendo que os da margem são maiores e mais evidentes. O hipocótilo é robusto, também com grande quantidade de glândulas de óleo essencial, inicialmente branco esverdeado com algumas porções levemente vináceas, tornando-se verde ao longo do seu desenvolvimento. O primeiro par de protófilos fica evidente a partir do $5^{\circ}$ e $6^{\circ}$ dia, eretos, verdes, opostos, simples, pedicelados, sem estípula, sem catáfilos e de margem inteira, nervura central bem evidente, com tricomas na margem e grande quantidade de glândulas de óleos essenciais, sendo mais evidentes aqueles da margem da lâmina foliar. A raiz emerge já apresentando pelos absorventes, desenvolvendo ramificações laterais entre $05^{\circ} \mathrm{e}$ $6^{\circ}$ dia após a germinação (Morgante et al., 2010).

Aemergência irregular e distribuída ao longo do tempo pode ser entendida como uma estratégia para aumentar a probabilidade de sobrevivência de alguns indivíduos, recurso muitas vezes utilizado pelas espécies silvestres (Gogosz, 2008).

\section{Usos e aplicações}

A família Myrtaceae é reconhecida por seu grande potencial na produção de óleos voláteis de interesse econômico (Lima et al., 2006). Registrase, na literatura, sua relevância frente às diversas ações de suas inúmeras espécies. Apesar disso, poucos são os estudos etnomedicinais, fitoquímicos e farmacológicos existentes sobre essa importante família (Aciole, 2009). Dentro dessa família, o gênero Pimenta tem sido bastante estudado devido às suas propriedades biológicas, que incluem atividade antimicrobiana, anti-inflamatória, analgésica, antipirética, antinociceptiva e hipotensiva, entre outras (Custódio et al., 2010; Paula et al., 2010a).

$P$. pseudocaryophyllus representa grande importância para o homem, sendo reconhecida para diversas finalidades no conhecimento tradicional. Medicinalmente, a espécie tem sido amplamente 
utilizada no tratamento de estados gripais, como calmante, regulador da digestão e menstruação (Nakaoka-sakita et al., 1994; Paula et al., 2008). Segundo a população local de Guaraqueçaba, PR, a infusão das folhas de P. pseudocaryophyllus combate artritismo, blenorragia, diarreias sanguíneas, disenteria, febres, sífilis, e possui atividade antihelmíntica. As folhas são utilizadas, ainda, como condimento no feijão e em carnes e ensopados (Oliveira et al., 2006). No Vale do Ribeira (SP) suas folhas são usadas pelas comunidades locais na forma de chá de fim diurético e para o combate de gripes, resfriados e fadiga. As folhas também são utilizadas para o preparo de inalações e solução alcoólica para massagens musculares (Morgante et al., 2010).

Outro uso popular das folhas de notada importância no litoral do estado do Paraná e São Paulo é na aromatização de aguardente, denominada "cataia", típica da região. Na região do Vale do Ribeira, a bebida é comercializada não somente em lojas de artesanato, mas também nos postos de abastecimento ao longo da rodovia BR 116 (Morgante et al., 2010). No estado do Paraná, a bebida foi produzida pioneiramente na comunidade de Barra do Ararapira, mas hoje é vendida em diversos vilarejos e centros urbanos da região (Bazzo, 2010). A bebida caracteriza-se com um produto de grande representatividade cultural e econômica para as comunidades da região (Morgante et al., 2010; Oliveira, 2010).

As bagas da espécie, depois de secas, são utilizadas como substituto ao cravo-da-índia em doces e geleias, conforme costume datado nos meados de 1800 (Lullez, 1991), devido à semelhança de cheiro e sabor dessas duas espécies (Farias, 2009). Desde o século XIX há correlações entre essas espécies, sendo que o próprio nome específico da planta remete à semelhança entre elas, pseudocaryophyllus significa algo como "falso cravo", dado que o nome científico do cravo-daindia é Caryophyllus aromaticus (Farias et al., 2009; Neves et al., 2009).

A madeira do craveiro é pesada (densidade $\left.1,00 \mathrm{~g} / \mathrm{cm}^{3}\right)$, dura, de textura fina a média, utilizada pelas pequenas dimensões disponíveis apenas localmente para obras internas em carpintaria, bem como para lenha e carvão. A árvore possui qualidades ornamentais que a recomendam para arborização urbana, principalmente para ruas estreitas e embaixo de redes elétricas. É recomendada também para reflorestamentos energéticos e preservacionistas (Lorenzi, 1998).

\section{Composição química do óleo essencial}

O gênero Pimenta apresenta como principal característica de seus óleos essenciais a ocorrência de éteresfenólicos como componentes majoritários, tais como eugenol, chavicol, estragol e metileugenol (Morgante et al., 2010).

A composição química completa do óleo essencial de P. pseudocaryophyllus, feita através de cromatografia gasosa acoplada à espectometria de massa, foi descrita pela primeira vez no ano de 1994 , identificando o geranial (34\%) e o neral (28\%), isômeros conhecidos como citral A e B, como compostos majoritários de amostra coletadas no Parque Estadual de Campos do Jordão, SP. Segundo os autores, tais componentes conferem às folhas aroma semelhante à cidreira (Nakaokasakita et al., 1994).

A extração do óleo essencial de $P$. pseudocaryophyllus coletada em Ilha Comprida (SP) revelou a presença de dezoito compostos, incluindo o eugenol e metil-eugenol como predominantes (Oliveira et al., 2006). O eugenol chegou a representar mais de $92 \%$ da composição do óleo, em espécimes coletados em Telêmaco Borba e São Jerônimo da Serra (PR) (Custódio et al., 2007; Custódio et al., 2010),

Citam-se outros compostos abundantes registrados na composição do óleo essencial desta espécie: geranial, neral, linalol, $\beta$-cariofileno, em espécimes coletados em Campos do Jordão (SP) (Nakaoka-sakita et al., 1994); geranial, neral, óxido de cariofileno, (E) metilisoeugenol, metileugenol, em espécimes coletados em São Gonçalo do Abaeté (MG) e Brasília (DF) (Paula et al., 2005); metileugenol, em espécimes coletados em Santo André (SP) e Brasília (DF) (Paula et al., 2005; Lima et al., 2006); (E)-metil isoeugenol, neral e geranial (cis-isômero e trans-isômero do citral) (Nakaoka-sakita et al., 1994); chavibetol, em espécimes coletados em llha Comprida, Cananéia, Ilha do Cardoso (SP), (Aciole, 2009; Marques et al., 2010; Santos, 2010); terpinen-4-ol, o-cimeno e (E)-cariofileno, nos espécimes coletados em São Jerônimo da Serra (PR) (Custódio et al., 2010).

Sabe-se que condições ambientais podem provocar variações significativas na produção de metabólitos secundários nos vegetais. Nesse sentido, Abaul e colaboradores (1995) descreveram três variedades da espécie $P$. racemosa, que se diferenciam quimicamente. Tais variedades são chamadas quimiotipos, uma vez que, taxonomicamente, são consideradas a mesma espécie, mas apresentam composição química distinta.

Estudos que analisam a composição química de $P$. pseudocaryophyllus apontam para a existência de diferentes quimiotipos para as diferentes populações encontradas no Brasil, assim como descrito para P. racemosa. Foram verificados indícios de variação nos teores de metabólitos secundários da matéria prima vegetal 
Tabela 1. Compostos majoritários encontrados no óleo essencial de $P$. pseudocaryophyllus em diferentes regiões do Brasil

\begin{tabular}{|c|c|c|}
\hline Referências & Local de coleta das folhas & Composto majoritário (percentual) \\
\hline $\begin{array}{l}\text { Nakaoka-Sakita et al. } \\
\text { (1994) }\end{array}$ & Campos do Jordão (SP) & Geranial $(34,3 \%)$ e neral $(27,8 \%)$ \\
\hline Custódio et al. (2007) & Telêmaco Borba (PR) & Eugenol $(92,8 \%)$ \\
\hline \multirow[t]{2}{*}{ Paula (2006) } & Brasília (DF) & (E)-metilisoeugenol $(78,0-93,6 \%)$ \\
\hline & São Gonçalo do Abaeté (MG) & Geranial $(36,56-47,2 \%)$ \\
\hline Custódio et al. (2010) & São Jerônimo da Serra (PR) & Eugenol $(92,6 \%)$ \\
\hline \multirow[t]{8}{*}{ Santos (2010) } & Ilha Comprida (SP) & \\
\hline & amostra 1, 2, 6 & Chavibetol $(68,6 \% ; 34,1 \% ; 32 \%)$ \\
\hline & Cananéia (SP) & \\
\hline & amostra 3 & Eugenol $(47,4 \%)$ \\
\hline & amostra 4 & Sesquiterpeno $(5,5)$ - $\alpha$ - farneseno $(15,8 \%)$ \\
\hline & amostra 5 & Chavibetol $(21,7 \%)$ \\
\hline & Ilha do Cardoso (SP) & \\
\hline & amostra 7 & Chavibetol $(26,2 \%)$ \\
\hline \multirow[t]{10}{*}{ Paula et al. (2011) } & São Gonçalo do Abaeté (MG) & \\
\hline & amostra $1,5,8$ & Geranial $(46,6 \% ; 39,6 \% ; 37,3 \%)$ \\
\hline & amostra $2,3,4$ & (E)-metilisoeugenol $(93,7 \% ; 61,3 \% ; 92,5 \%)$ \\
\hline & amostra 5 & (E) cariofileno $(26,6 \%)$ \\
\hline & amostra 7 & (E)-metilisoeugenol $(91,5 \%)$ \\
\hline & São José do Barreiro (MG) & \\
\hline & amostra 1,2 & (E)-metilisoeugenol (94,3\%; 93,8\%) \\
\hline & amostra 3 & $(E)$-asarona \\
\hline & Brasília (DF) & \\
\hline & amostra 1 & (E)-metilisoeugenol $(93,6 \%)$ \\
\hline
\end{tabular}

de $P$. pseudocaryophyllus relacionada a fatores fenológicos e geográficos. (Paula et al., 2008).

Paula et al. (2011) avaliaram os componentes do óleo essencial de folhas de doze espécimes de $P$. pseudocaryophyllus coletadas nos municípios de São Gonçalo do Abaeté (MG), São José do Barreiro (MG) e Brasília (DF). Foram verificados 57 constituintes e a analise dos componentes principais
(PCA) e de agrupamento (CA) identificou três quimiotipos, sendo eles: grupo I, caracterizados por um elevado percentagens de geranial, neral, óxido de cariofileno e espatulenol; grupo II, com elevados teores de (E)-asarona, (E)-cariofileno e elemicim; e o grupo III, com quantidades elevadas de (E)-metil isoeugenol. Segundo os autores, a ocorrência dos diferentes quimiotipos no mesmo local indica que 
variação química é geneticamente determinada.

Os dados descritos na Tabela 1 demonstram a variabilidade de compostos majoritários encontrados no óleo essencial de amostras da espécie coletadas em diferentes regiões do país.

\section{Potencial farmacológico}

$P$. pseudocaryophyllus apresenta composição química complexa, tendo como característica a presença de compostos fenólicos, taninos e flavonoides, o que sugere importante potencial fitoterapêutico a ser investigado, além de apresentar traços de heterosídeos antraquinônicos (Paula et al., 2008; El Assal, 2012).

A atividade antimicrobiana e antifúngica do extrato obtido de folhas de $P$. pseudocaryophyllus tem sido amplamente testada.

Chegando a representar $92 \%$ da composição do óleo essencial das folhas da espécie, o eugenol é apontado como o principal responsável pelas propriedades antissépticas que a espécie apresenta (Farias et al., 2009). Registram-se diversas finalidades do eugenol na indústria, incluindo flavorizante em produtos alimentícios, especialmente em carnes e salsichas, sendo também usado em condimentos. É também usado na fabricação de perfumes, cosméticos e sabonetes, também servindo de matéria prima para a produção do isoeugenol, que por sua vez é usado na fabricação da vanilina (baunilha) (Lullez, 1991). $O$ eugenol foi identificado, ainda, em 31 marcas de whiskies de vários países a partir da análise por cromatografia gasosa (Lehtonen, 1982).

Assim como se verificam indícios de variabilidade nos teores de metabólitos secundários de P. pseudocaryophyllus relacionada a fatores fenológicos e geográficos, também se observa diferentes padrões de atividade antimicrobiana (Paula et al., 2010a).

Lima et al. (2006), em estudo em que se consideraram populações de P. pseudocaryophyllus coletadas em dois ecossistemas distintos, observaram que os óleos essenciais das diferentes populações tinham composição química diferente, assim como diferente sensibilidade a determinadas bactérias. O espécime coletado na llha do Cardoso (SP) teve os melhores resultados contra E. coli; frente a $P$. aeruginosa e a $S$. aureus os espécimes mostraram resultados similares; e frente a $C$. albicans o espécime coletado no Parque Municipal Nascentes do Paranapiacaba, em Santo André (SP) apresentou resultados mais expressivos.

Paula et al. (2012) avaliaram a atividade antimicrobiana do extrato bruto de etanol, frações e substâncias semipurificadas deste, e de óleos essenciais obtidos a partir de folhas dos dois quimiotipos mais frequentemente encontrados na região de coleta (São Gonçalo do Abaeté, MG), quimiotipo (E)-metil isoeugenol e quimiotipo citral. Testou-se a bioatividade contra as bactérias Gram positivas Staphylococcus sp., Micrococcus sp., e Bacillus sp., bactérias Gram negativas Escherichia coli, Enterobacter sp., Serratia marcescens, Salmonella spp. e Pseudomonas aeruginosa, e contra os fungos Candida sp. e Cryptococcus sp. Entre as substancias isoladas, o óleo oleanólico obtido de uma fração do extrato etanólico do quimiotipo citral apresentou os melhores resultados (MIC $=31.2$ to $125 \mu \mathrm{gmL}-1$ ) contra as bactérias Gram positivas analisadas, seguido pelo óleo essencial do mesmo quimiotipo, que mostrou boa atividade (MIC $=62.5 \mathrm{~g} \mu \mathrm{gmL}-1$ ) contra $B$. cereus e atividade moderada ( $\mathrm{MIC}=125-250 \mu \mathrm{gmL}-1$ ) contra as demais bactérias Gram positivas. A atividade antifúngica apresentada pelo acetato de etila e pela fração aquosa dos dois quimiotipos, especialmente contra Candida albicans (MIC $=31.2$ to $62.5 \mu \mathrm{gmL}-1$ ) e C. parapsilosis (MIC $=15.6$ to 62.5 $\mu \mathrm{gmL}-1$ ), demonstram o potencial da espécie como fonte de novas alternativas antifúngicas.

Avaliou-se, ainda, a atividade antiinflamatória e antinoceptiva de extratos e frações em diferentes doses, pelos testes de edema de orelha induzido por óleo de Croton e teste de contorção abdominal induzida pelo ácido acético, respectivamente. $\mathrm{O}$ extrato cru de etanol do quimiotipo citral mostrou efeitos anti-inflamatórios e antinociceptivo, o que, segundo os autores, pode estar relacionado à presença de triterpenos pentacíclicos lupeol, $\alpha$-amirina, e $\beta$-amirina e os flavonóides quercetina, quercitrina, e afzelina.

O óleo essencial de espécimes coletados no Parque Estadual de Campos do Jordão (SP) apresentou atividade frente os fungos Aspergillus niger e Penicillium verrucosum, e contra as bactérias Escherichia coli e Staphylococcus aureus (Sakita E Aguiar, 2007). Em estudo realizado na região de Telêmaco Borba (PR) constatou-se atividade contra Alternaria sp., Botryosphaeria ribis, Botryosphaeria sp. e Aspergillus niger. O ensaio de atividade antifúngica por autobiografia em cromatoplaca apontou o eugenol como o responsável por tal atividade (Custódio et al., 2007).

Com o propósito de encontrar a substância responsável pela atividade do óleo essencial de P. pseudocaryophyllus, Santos (2010) avaliou a atividade antifúngica do chavibetol, metileugenol e eugenol, extraídos de espécimes coletados em Ilha Comprida, Cananéia e llha do Cardoso (SP). A análise dos resultados mostrou o chavibetol como responsável pela atividade antifúngica deste óleo frente às cepas de Trichophyton spp. e Microsporum spp (Santos, 2010).

Oliveira e colaboradores (2006) registraram 
atividade antiparasitária "in vitro" do óleo essencial de $P$. pseudocaryophyllus contra promastigotas de Leishmania chagasi a uma concentração de $500 \mu \mathrm{g} / \mathrm{ml}$ observando-se mortandade de $100 \%$. Registraram-se também excelentes resultados quanto ao potencial ativo do óleo essencial da espécie sobre as larvas de $A$. aegypti (Barreto et al., 2006; Aciole, 2009).

O extrato aquoso de P. pseudocaryophyllus possui significativo efeito de repelência sobre o adulto da broca de bananeira (Cosmopolites sordidus) (Pavarini et al., 2010). O extrato etanólico de P. pseudocaryophyllus não apresentou atividade inseticida promissora para uso no manejo de Sitophilus zeamais (Ansante et al., 2011).

A administração oral do extrato etanólico de $P$. pseudocaryophyllus em camundongos não apresentou qualquer sinal de neurotoxicidade, mas sugere uma atividade de depressão do sistema nervoso central, enquanto que os tratamentos com fração de diclorometano e com fração aquosa, também obtidos das folhas da espécie, foram caracterizados por efeitos ansiolítico e sedativo, respectivamente. (Fajemiroye et al., 2012).

Está sendo investigado o efeito do óleo essencial de P. pseudocaryophyllus na promoção da permeação cutânea, que propicia a veiculação de fármacos através da pele em formulações tópicas. $O$ estudo visa avaliar a eficácia do óleo dessa espécie e de seus componentes majoritários na permeação in vitro do diclofenaco de sódio utilizando membrana natural e biológica artificial (Kaneko, 2011).

Está em andamento pesquisa com $P$. pseudocaryophyllus que inclui a avaliação da citotoxicidade, da fototoxicidade e do potencial genotóxico (Kaneko \& Pedro, 2010).

\section{Rendimento de óleo}

Detectou-se variação no rendimento de óleo essencial das folhas de P. pseudocaryophyllus colhidas no Parque Estadual de Campos do Jordão (SP) em diferentes meses do ano. Verificou-se que em outubro, novembro e maio o rendimento foi mais baixo. Os melhores rendimentos foram obtidos de janeiro a abril (Sakita \& Aguiar, 2007). Em estudo realizado com espécimes de Brasília (DF) e São Gonçalo do Abaeté (MG), o rendimento foi diferenciado em distintas fenofases, obtendo-se maior rendimento nos períodos pré e pós- antese $(0,8 \%)$ (Paula et al., 2010b).

Lullez (1991) obteve rendimento de 1\% em espécimes coletados no estado do Paraná. Em estudo realizado no mesmo estado, na região de Tijucas do Sul, Girard e colaboradores obtiveram um rendimento médio do óleo essencial (base folhas secas) de 2,64\%, com variação de 2,42 a 3,02\% (Girard et al., 2007).

\section{CONSIDERAÇÕES FINAIS}

A partir da revisão bibliográfica de Pimenta pseudocaryophyllus conclui-se que a espécie tem grande valor do ponto de vista medicinal, além de representar importância cultural e econômica. Uma maior investigação a respeito das propriedades medicinais da espécie que inclua estudos de sua toxicidade e segurança é necessária, uma vez que a utilização racional de plantas com fins terapêuticos é de grande valor no setor de saúde em países em desenvolvimento. O óleo essencial da planta, de atividade antimicrobiana e antiparasitária comprovada, tem potencial para o desenvolvimento de novas substâncias. Por fim, nota-se uma carência de informações a respeito da fenologia, assim como informações ecológicas de forma geral.

\section{REFERÊNCIA}

ACIOLE, S.D.G. Avaliação da atividade inseticida dos óleos essenciais das plantas amazônicas Annonaceae, Boraginaceae e de Mata Atlântica Myrtaceae como alternativa de controle às larvas de Aedes aegypti (Linnaeus, 1762) (Diptera: Culicidae). 2009. 86p. Dissertação (Mestrado em Biologia Humana e Ambiente) - Faculdade de Ciências, Universidade de Lisboa, Lisboa.

AGRA, M. F. Contribuição ao estudo das plantas medicinais na Paraíba. Ciência e Cultura, v.33, p. 64-66. 1982.

AMOROZO, M.C.de.M. Uso e diversidade de plantas medicinais em Santo Antonio do Leverger, MT, Brasil. Acta Botanica Brasilica, vol.16, n.2, p.189-203, 2002.

ANSANTE, T.F. et al. Extrato etanólico de Pimenta pseudocaryophyllus (Myrtaceae): ação inseticida e no comportamento de seleção hospedeira de Sitophilus zeamais Mots. (Col.: Curculionidae). In: SIMPÓSIO INTERNACIONAL DE INICIAÇÃO CIENTÍFICADA USP, 19, São Paulo, 2011. Anais...2011.

BANDEIRA, F. P. S. de F. Prefácio. In: ALBUQUERQUE, U. P. de; LUCENA, R. F. de P.; CUNHA, L. V. F. C. da. Métodos e Técnicas na Pesquisa Etnobiológica e Etnoecológica. Recife: NUPPEA, 2010.

BARRETO, C.F. et al. Estudo das alterações morfohistológicas em larvas de Aedes aegypti (Diptera, Culicidae) submetidas ao extrato bruto etanólico de Sapindus saponária Lin. (Sapindaceae). Revista de Patologia Tropical, v.35, n.1, p. 37-57, 2006.

$B A Z Z O, J$. Mato que vira mar, mar que vira mato: 0 território em movimento na vila de pescadores da Barra de Ararapira (Ilha do Superagui, Guaraqueçaba, Paraná). 2010. 291 p. Dissertação (Mestrado em Antropologia Social) - Setor de Ciências Humanas, Letras e Artes, Universidade Federal do Paraná, Curitiba.

BRANDÃO, M. Árvores nativas do estado de Minas Gerais. Belo Horizonte: EPAMIG. 2002.

CUSTÓDIO, D.L. et al. Antimicrobial activity of essential oils from Pimenta pseudocaryophyllus and Tynanthus micranthus. Brazilian Archives of Biology and 
Technology. v.53, n.6, p.1363-1369, 2010.

CUSTÓDIO, D. L. et al. Estudo do óleo essencial de Pimenta pseudocaryophyllus (Gomes) L. R. Landrum. In: SIMPÓSIO BRASILEIRO DE ÓLEOS ESSENCIAIS, 4, 2007, Fortaleza. Anais...2007.

EL ASSAL, F.E. Bioatividade in vitro de Pimenta pseudocaryophyllus sobre Candida spp e complexo Cryptococcus neoformans. 2012. 85f. Tese (Doutorado em Medicina Tropical e Saúde Pública) - Universidade Federal de Goiás.

FARIAS, V.De et al. Organização estrutural da folha de Pimenta pseudocaryophyllus (Gomes) L.R. Landrum, Myrtaceae. Acta Botanica Brasilica, v.23, n.2, p.398406, 2009.

FARNSWORTH, N. R. Screening plants for new medicines. In: Wilson, E.O. Biodiversity. Washington DC: Nac. Acad. Press, 521p. 1988

FAJEMIROYE, J.O. et al. Central activities of Pimenta pseudocaryophyllus (Gomes) L.R. Landrum. Central Intitute of Medicinal and Aromatic Plants, v.2, n.1, p.118-122, 2012.

GIRARD, E.A. Volume, biomassa e rendimento de óleos essenciais do craveiro (Pimenta pseudocaryophyllus (Gomes) Landrum) em Floresta Ombrófila Mista. 2005. 60p. Dissertação (Mestrado em Ciências Florestais) - Universidade Federal do Paraná, Curitiba.

GIRARD, E.A.; KOEHLER, H.S.; NETTO, S.P. Volume, biomassa e rendimento de óleos essenciais do craveiro (Pimenta pseudocaryophyllus (Gomes) Landrum). Revista Acadêmica, v.5, n.2, p.147-165, 2007.

GOGOSZ, A.M. Germinação e estrutura das plântulas de Campomanesia xanthocarpa O. Berg. (Myrtaceae) crescendo em solo contaminado com petróleo e solo biorremediado. 2008. 100 p. Dissertação (Mestrado em Botânica) - Setor de Ciências Biológicas, Universidade Federal do Paraná, Curitiba.

GRESSlER, E.; PIZO, M. A.; MORELLATO, L. P. C. Polinização e dispersão de sementes em Myrtaceae do Brasil. Revista Brasileira de Botânica, v. 29, n.4, p.509-530, 2006.

KANEKO, T.M. Avaliação do óleo essencial da espécie nativa de Pimenta pseudocaryophyllus (Gomes) Landrum como promotor de permeação cutânea utilizando membrana natural e biológica artificial. Universidade de São Paulo. Faculdade de Ciências Farmacêuticas (Fundação de Amparo à Pesquisa do Estado de São Paulo, Projeto 2010/20533-5). Projeto em andamento. 2011.

KANEKO, T.M. \& PEDRO, N.M.E. Avaliação in vitro da toxicidade de óleos essenciais da flora latinoamericana candidatos ao uso em cosméticos. Universidade de São Paulo. Faculdade de Ciências Farmacêuticas (Fundação de Amparo à Pesquisa do Estado de São Paulo, 10/03053-0). Projeto em andamento. 2010.

LANDRUM, L.R. Campomanesia, Pimenta, Blepharocalyx, Legrandia, Acca, Myrrhinium and Luma (Myrtaceae). Flora Neotropica Monograph, v.45, p.1-179, 1986.

LEGRAND, D.L.; KLEIN, R.M. Mirtáceas. In: REITZ, R. Flora ilustrada catarinense. Itajaí: Herbário Barbosa Rodrigues, 1978, p.572-730.

LEHTONEN, M. Phenols in Whisky. Chromatographia, v.16, p.201-203, 1982.
LIMA, M.E.L. et al. Antimicrobial activity of the essential oil from two specimens of Pimenta pseudocaryophyllus (Gomes) L. R. Landrum (Myrtaceae) native from São Paulo state - Brazil. Pharmacologyonline, v.3, p.589593, 2006.

LORENZI, H. Árvores brasileiras: manual de identificação e cultivo de plantas arbóreas nativas do Brasil. 2. ed., vol.2, Nova Odessa: Instituto Plantarum, 1998. 352 p.

LORENZI, H. Árvores brasileiras: manual de identificação e cultivo de plantas arbóreas nativas do Brasil. 2.ed. Nova Odessa: Instituto Plantarum, 2002. 382p.

LULLEZ, J.C. Identificação de metileugenol, eugenol e chavibetol no óleo essencial da folha de Pseudocaryophyllus acuminatus (Limk) Burret. 1991. 124 p. Dissertação (Mestrado em Engenharia Química) - Universidade Federal do Paraná, Curitiba.

MARQUES, F.A. et al. Leaf essential oil composition of Pimenta pseudocaryophyllus (Gomes) L. R. Landrum Native from Brazil. Journal of Essential Oil Research, v.22, n.2, p.150-152, 2010.

MAZINE, F.F.; SOUZA, V.C. Myrtaceae dos campos de altitude do Parque Nacional do Caparó - Espírito Santo/ Minas Gerais, Brasil. Rodriguésia, vol.59, n.1, p.57-74, 2008.

MISSOURI BOTANICAL GARDEN. Disponível em: <http:// www.tropicos.org/Name/22103938>. Acesso em: 6 mar. 2012.

MORGANTE, P.G. et al. Cataia: muito consumida, pouco conhecida. In: SILVA, R.B.da; MING, L.C. Polo de Biotecnologia da Mata Atlântica: Relatos de pesquisas e outras experiências vividas no Vale do Ribeira. Jaboticabal: Maria de Lourdes Brandel - ME, 2010, p.20-40.

NAKAOKA-SAKITA et al. Óleo essencial de Pimenta pseudocaryophyllus var. pseudocaryophyllus (Gomes) Landrum (Myrtaceae) I: cromatografia a gás/espectrometria de massa (CG/ EM). Revista do Instituto Florestal, v.6, p.53-61, 1994.

NEVES, I. de A. et al. Composição química e atividade acaricida do óleo essencial do cravo-da-india (Caryophyllus aromaticus L.). In: JORNADA DE ENSINO PESQUISA E EXTENSÃO, 2009, Recife. Anais... 2009.

OLIVEIRA, A.F.de. Avaliação fitoquímica dos extratos de folhas de Pimenta pseudocaryophyllus (cataia). 2010. 44p. Monografia (Bacharel em Gestão Ambiental) - Setor Litoral, Universidade Federal do Paraná, Matinhos.

OLIVEIRA, C.A.; PRADO, J.E.; GUERRERO JUNIOR, P.G. 2006. Composição química e utilização de metabólitos voláteis de Pimenta pseudocaryophyllus. In: CONGRESSO DE INICIAÇÃO CIENTÍFICADA UNESP, 18, 2006, Jaboticabal. Anais... São Paulo: CGB/PROPE UNESP, 2006.

PAULA, J.A.M. Estudo farmacognóstico e avaliação da atividade antimicrobiana das folhas de Pimenta pseudocaryophyllus (Gomes) L. R. Landrum Myrtaceae. 2006. 139p. Dissertação (Mestrado em Biologia) - Instituto de Ciências Biológicas, Universidade Federal de Goiás, Goiânia.

PAULA, J.A.M. et al. Estudo farmacognóstico das folhas 
de Pimenta pseudocaryophyllus (Gomes) R. Landrum - Myrtaceae. Revista Brasileira de Farmacognosia, v.18, n.2, p.265-278, 2008

PAULA, J.A.M. et al. Antimicrobial activity of the crude ethanol extract from Pimenta pseudocaryophyllus. Pharmaceutical Biology, v. 47, n.10, p. 987-993, 2009.

PAULA, J.A.M. et al. Gênero Pimenta: aspectos botânicos, composição química e potencial farmacológico. Revista Brasileira de Plantas Medicinais, v.12, n.3, p.363-379, 2010a.

PAULA, J.A.M. et al. Chemical Differences in the Essential Oil of Pimenta pseudocaryophyllus (Gomes) L. R. Landrum Leaves from Brazil. Journal of Essential Oil Research, v.22, n.6, p.555-557, 2010b.

PAULA, J.A.M. et al. Infraspecific chemical variability in the essential oils of Pimenta pseudocaryophyllus (Gomes) L.R. Landrum (Myrtaceae). Biochemical Systematics and Ecology, vol.39, p.643-650, 2011.

PAULA, J.A.M. et al. Phytochemical Analysis and Antimicrobial, Antinociceptive, and Anti-Inflammatory Activities of Two Chemotypes of Pimenta pseudocaryophyllus (Myrtaceae). Evidence-Based Complementary and Alternative Medicine. 2012.

PAVARINI, R.; ANSANTE, T.F.; CACERES, D.P. Efeito do extrato aquoso de plantas sobre a broca da bananeira Cosmopolites sordidus Germar (Coleoptera: Curculionidae). Nucleus, v.7, n.1, p.203-208, 2010.

PÉLLICO NETTO, S.; STERMER, R.P.; WEBER, S.H. Análise da regeneração natural do craveiro, Pimenta pseudocaryophyllus (Gomes) Landrum. Revista Acadêmica, v.5, n.2, p.131-137, 2007.

RUSCHEL, A.R. Pimenta pseudocaryophyllus. In: CORADIN, L.; SIMINSKI, A.; REIS, A. Espécies nativas da flora brasileira de valor econômico atual ou potencial: plantas para o futuro - Região Sul. Brasília: MMA, 2011, p. 223-225.

SANTOS, B.C.B.dos. Fitoquímica e ensaios biológicos de óleos essenciais de Pimenta pseudocaryophyllus e Hedychium coronarium da Mata Atlântica do Estado de São Paulo. 2010. 92 f. Dissertação (Mestrado em Química na área de Química Orgânica) - Universidade Estadual de Campinas, Campinas.

SEBASTIÃO, I. et al. 2011. Marcadores moleculares microssatélites isolados de Pimenta pseudocaryophyllus (Gomes) Landrum, Myrtaceae. In: CONGRESSO DE INICIAÇÃO CIENTÍFICA DA UNESP, 2011, São Pedro. Anais... 2011.

SOBRAL, M. et al. Myrtaceae. In: Lista de espécies da flora do Brasil. Rio de Janeiro: Jardim Botânico do Rio de Janeiro, 2010. Disponível em: <http://floradobrasil. jbrj.gov.br/jabot/floradobrasil/FB171>. Acesso em: 12 mar. 2012

SOUZA, V. C.; LORENZI, H. Botânica Sistemática - Guia ilustrado para identificação das novas famílias de angiospermas da flora brasileira, baseado em APG II. $2^{\mathrm{a}}$ ed. Nova Odessa, SP: Instituto Plantarum, 2008. $640 p$.

STAGGEMEIER, V.G.; MORELLATO, L.P.C.; GALETTI, M. Fenologia reprodutiva de Myrtaceae em uma ilha continental de Floresta Atlântica. Revista Brasileira de Biociências, Porto Alegre, v.5, n.1, p.423-425, 2007.

WEISS, E.A Spice Crops. New York: CABI, 2002. 399p. WORLD HEALTH ORGANIZATION. Traditional medicine and modern health care. Geneva: WHO,1991. 